See discussions, stats, and author profiles for this publication at: https://www.researchgate.net/publication/265165713

\title{
Wear Prediction of Ceramic-on-Ceramic Artificial Hip Joints
}

Chapter · August 2014

CITATIONS

4

4 authors:

Ehsan Askari

54 PUBLICATIONS 649 CITATIONS

SEE PROFILE

Danè Dabirrahmani

Macquarie University

44 PUBLICATIONS 373 CITATIONS

SEE PROFILE

Some of the authors of this publication are also working on these related projects:

Numerical study of pulsatile blood flow in a bifurcation View project

Biomechanics View project
READS

159

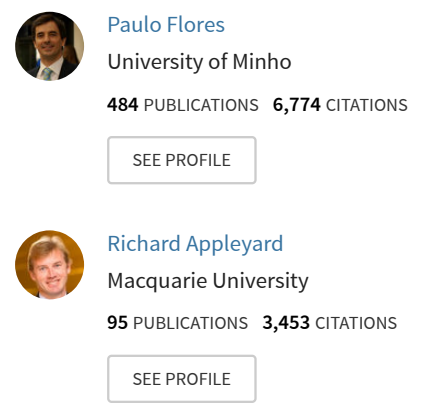




\title{
Wear prediction of ceramic-on-ceramic hip artificial joints
}

\author{
E. Askari ${ }^{1}$, P. Flores $^{2}$, D. Dabirrahmani ${ }^{3}$ and R. Appleyard ${ }^{4}$ \\ ${ }^{I}$ Macquarie University, Australia,e-mail: ehsanaskary@gmail.com \\ ${ }^{2}$ University of Minho, Portugal, e-mail: pflores@dem.uminho.pt \\ ${ }^{3}$ Macquarie University, Australia, e-mail: daneh.turner@mq.edu.au \\ ${ }^{4}$ Macquarie University, Australia, e-mail: richard.appleyard@mq.edu.au
}

\begin{abstract}
Wear can influence the lifetime and performance of implants and has been found to be a key factor in primary failure of artificial hip joints. The present study aims to present a spatial multibody dynamic model to predict wear in ceramic-on-ceramic hip implants. The problem was formulated by developing a spatial multibody dynamic model of a hip prosthesis taking three-dimensional physiological loading and motion of the human body into account. Then, the Archard wear model was integrated into the dynamic calculation of the hip implant to predict wear. Additionally, geometries of the cup and head were updated throughout the simulation to generate a more realistic wear simulation. The results were validated against current literature. Finally it was illustrated that friction-induced vibration caused excessive wear of hip implant components.
\end{abstract}

Key words: Wear simulation, ceramic-on-ceramic hip joint, multibody systems.

\section{Introduction}

Friction-induced vibration is an undesirable oscillation in artificial hip joints due to tribological interactions between the head and cup. These tribological interactions during the human daily activities can cause articulating surfaces of hip implants to undergo wear. Moreover, vibration induced by friction can lead to excessive wear of the head and cup of hip implants [1]. Wear can influence the lifetime and performance of implants and has been found to be a key factor in primary failure of artificial hip joints [2].

The most commonly used artificial hip joint combination is a metal head within a plastic cup which is referred to a soft-on-hard couple. This combination is known to suffer from cup wear with the resultant plastic debris reported to induce osteolysis. Computing the wear profile of soft-on-hard bearing couples has been the subject of a number of studies [3-4]. In an effort to reduce wear, hard-on-hard material combinations have been developed namely metal-on-metal (MoM) and 
ceramic-on-ceramic $(\mathrm{CoC})$. However with hard-on-hard couples, wear occurs across both the head and cup surface [4]. Ceramic-on-Ceramic hip implants have been used increasingly since their introduction in the 1970s [5]. Ceramic bearings have the potential advantage of lower wear rates in total hip arthroplasty compared with metal-on-metal hip implants and soft-on-hard bearings [6]. Despite very low wear rates of ceramic bearings, it continues to be an important research topic because wear of artificial hip joints is a key issue leading to their primary failure even in $\mathrm{CoC}$ hip arthroplasties.

To evaluate implant wear computationally, Archard's wear model is the most commonly utilised method [4]. This wear model requires knowledge of the contact pressure, sliding distance of the contact point and tribological data such as the wear coefficient of the bearing materials. Implant head-cup contact properties can be numerically determined by means of the finite element method [7], boundary element method [2] and Hertz contact model [8].

The contact point between the head and cup follows a certain characteristic track during normal human gait, and this 'contact point track' is an important parameter to determine wear since any variation in the track shape can cause a huge variation in the wear rate [9]. Mattei et al. [10] used a theoretical contact point track, where the contact point was assumed to be located at the interface of the head and cup and along the line joining both the centres of the head and cup. Another study assumed that the centre of the femoral head was fixed and the motion of the femur was simulated by applying physiological rotations [3]. Furthermore, Saikko and Calonius [11] developed a computational method based on Euler angles to compute the slide tracks for the three-axis motion of the hip joint in walking and for two hip simulators. The slide track patterns resulting from the gait waveforms were found to be similar to those produced by hip simulators. Sariali and his co-workers [12] also provided sliding path of motion between the head and cup when the hip implant is in edge-loading or in normal centred conditions using the Leeds II hip simulator.

Recently it was reported that, on top of the normal gross motion, the femoral head vibrates inside the cup with micron amplitude due to friction-induced vibration [13-14]. This results in a change in the contact point trajectory in both micro and macro scales, which can affect the final wear profile. Mattei and Di Puccio [15] recently determined the changes of contact point trajectory due to friction and calculated its effect on wear prediction. However, their model did not include the vibration of the femoral head inside the cup and they reported that wear decreased as friction increased which cannot be justified physically. To the best of our knowledge, no one has investigated the effect of friction-induced vibration on both wear prediction and the corresponding wear map of artificial hip joints.

Thus, the influence of friction-induced vibration on wear simulation of ceramicon-ceramic artificial hip joints is modeled and studied in the present investigation. Moreover, it is hypothesised that this undesirable oscillation can lead to excessive wear in artificial hip joints. For this purpose, a spatial multibody dynamic model is developed, which allows for the evaluation of both sliding distance and contact pressure in order to calculate the wear of hip implants. The friction-induced vibration and contact-impact events occurring between the head and cup surfaces are 
taken into consideration as external generalised forces in the governing equation of the motion. A friction-velocity relation and a Hertz contact model are employed to formulate tangential and normal contact forces, respectively. Three-dimensional physiological loading and motion of the human body are also taken into account. Nonlinear governing motion equations are solved, using adaptive Runge-KuttaFehlberg method and the geometry of worn bearing surfaces is updated during the wear simulation. Finally, the methodology is validated by comparing the wear prediction to current literature. Moreover, the influence of friction on wear predictions and the corresponding wear maps are studied.

\section{Governing motion equations of multibody systems}

It is well known that the equations of motion for a multibody dynamic system with holonomic constraints can be written as [20],

$$
\begin{aligned}
& \mathbf{M} \ddot{\mathbf{q}}=\mathbf{g}+\mathbf{g}^{(c)} \\
& \boldsymbol{\Phi}(\mathbf{q}, t)=0
\end{aligned}
$$

in which $\mathbf{M}$ is the system mass matrix, $\mathbf{q}$ generalized coordinates of the system, $\ddot{\mathbf{q}}$ the acceleration vector, and $\mathbf{g}$ the generalized force vector containing all external forces and moments. The bodies in the multibody system are interconnected by joints imposing constraints on the bodies' relative motion. Expressing these conditions as algebraic equations in terms of a generalised coordinate and time, $t$, holonomic kinematic constraints defined in Eq. (2) are introduced. Moreover, $\mathbf{g}^{(c)}$ is the vector of constraint reaction equations, which can be rewritten by means of the Jacobian matrix of the constraint equations $\left(\boldsymbol{\Phi}_{\mathbf{q}}\right)$ and the vector of Lagrange multipliers $(\boldsymbol{\lambda})$ as [16]

$$
\mathbf{g}^{(c)}=-\Phi_{q}^{T} \lambda
$$

Substituting Eq. (3) in Eq. (1) yields,

$$
\mathbf{M} \ddot{\mathbf{q}}+\boldsymbol{\Phi}_{\mathbf{q}}^{T} \lambda=\mathbf{g}
$$

Furthermore, differentiating Eq. (2) twice with respect to time, the constraint equation can be written as follows 


$$
\boldsymbol{\Phi}_{\mathbf{q}} \ddot{\mathbf{q}}=-\left(\boldsymbol{\Phi}_{\mathbf{q}} \dot{\mathbf{q}}\right)_{\mathbf{q}} \dot{\mathbf{q}}-2 \Phi_{\mathbf{q}} \dot{\mathbf{q}}-\Phi_{t t}=\gamma
$$

where $\gamma$ is a vector function of velocity and position of the system as well as time. As a consequence, both Eq. (4) and (5) yield a system of differential algebraic equations to be solved for $\ddot{\mathbf{q}}$ and $\boldsymbol{\lambda}$, given by

$$
\left[\begin{array}{cc}
\mathbf{M} & \boldsymbol{\Phi}_{\mathbf{q}}^{T} \\
\boldsymbol{\Phi}_{\mathbf{q}} & 0
\end{array}\right]\left\{\begin{array}{l}
\ddot{\mathbf{q}} \\
\lambda
\end{array}\right\}=\left\{\begin{array}{l}
\mathbf{g} \\
\gamma
\end{array}\right\}
$$

The equation (6) can be solved only if the coefficient matrix of Eq. (6) is nonsingular. This can be achieved by having a positive definite mass matrix and the Jacobian matrix $\boldsymbol{\Phi}_{\mathbf{q}}$ full row rank [13].

\section{Numerical wear models}

Wear is the progressive loss of substance as a result of relative motion between surfaces. Although some typical features of the wear process are demonstrated experimentally and there are some simple models to compute wear, such as the wellknown Archard's wear methodology, no laws of wear exist. The Archard wear equation can be written as follow

$$
I=\frac{V}{S}=\frac{K P}{H}
$$

where the dimensionless constant $K$ denotes the wear coefficient, $V$ is volumetric wear, $s$ sliding distance, $P$ contact pressure and $H$ is material hardness. Moreover, it is more preferable for engineering applications to utilise the ratio $K / H$, termed wear coefficient $(k)$, dimensionally expressed as $\mathrm{mm}^{3} / \mathrm{Nm}^{-1}$. In addition to the volumetric wear model of Archard defined in Eq. (7), the Archard's depth wear equation as the linear wear rate can be written as follows

$$
\frac{\mathrm{d} h}{\mathrm{~d} s}=\frac{K P}{H}
$$

in which $P$ is contact pressure and $h$ is depth wear.

A numerical solution for the wear depth, using Archard's wear model Eq. (8), may be obtained by the forward Euler integration algorithm to yield the following updating formula for wear depth 


$$
h_{i}=h_{i-1}+k P_{i} \Delta s_{i}
$$

in which $h_{i}$ refers to the wear depth at the $i$ th step while $h_{i-1}$ represents the wear depth at the previous step. The last term in Eq. (9) is the incremental wear depth which is a function of the contact pressure and the incremental sliding distance at the corresponding cycle.

\section{Results and Discussion}

The main objective of this study is to investigate the wear of CoC artificial hip joints with the following geometric properties: the femoral head radius equal to 14 $\mathrm{mm}$ and radial clearance of $50 \mu \mathrm{m}$. Material parameters of the bearing couples were defined according to Uddin and Zhang [17]. $\mathrm{Al}_{2} \mathrm{O}_{3}$ ceramic had a Young's modulus and Poisson ratio of $375 \mathrm{GPa}$ and 0.3 , respectively. The wear factor for CoC couples was assumed to be $0.2 \times 10^{-8}$ [17]. In vivo three dimensional physiological loads and rotations were applied to the femoral head centre and nonlinear dynamic equations were solved numerically.

Table 1 lists the results from the current study and compares them with the available literature. It can be seen that linear and volumetric wear results obtained from the present work compare well with hip simulator and computational studies as well as clinical studies.

Table 1. Comparison of results with the available literature.

\begin{tabular}{|c|c|c|c|}
\hline & Method & Linear wear $(\mu \mathrm{m})$ & Volumetric wear (mm3) \\
\hline $\begin{array}{l}\text { Present study (very low } \\
\text { friction) }\end{array}$ & Computation & 2.1834 & 0.16296 \\
\hline \multirow[b]{2}{*}{ Present study (friction) } & \multirow[b]{2}{*}{ Computation } & $0.1015 \mathrm{~mm}$ (cup) & 6.836 \\
\hline & & $\begin{array}{l}0.1860 \mathrm{~mm} \\
\text { (head) }\end{array}$ & \\
\hline $\begin{array}{c}\text { Dorlot [18]; } \\
\text { Mittelmeier and Heisel, } \\
{[19]}\end{array}$ & Clinical tests & $2-5$ & 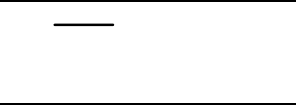 \\
\hline $\begin{array}{l}\text { Stewart et al. [20], } \\
\text { Affatato et al. [21] }\end{array}$ & Hip simulator & - & $0.1-1.015$ \\
\hline Uddin and Zhang [17] & Computation & 1.317 & 0.17275 \\
\hline Affatato et al. [22] & Clinical tests & $\begin{array}{l}0.014-1.4 \mathrm{~mm} \\
\text { (head) }\end{array}$ & $0.34-695$ \\
\hline
\end{tabular}

Trajectory and linear wear computation of the cup and femoral head for CoC hip implants are depicted in Fig. 1. The trajectories of the contact point between the cup and head conform to the worn path both in terms of location and shape. Moreover, the present study determined that wear increases as bearing surface 
friction increases. This conclusion is depicted in Fig. 1 where linear and volumetric wear of the friction model were around 50 times greater than the case with very low friction. This wear phenomenon can be justified by considering two physical reasons; (i) friction induced an increase in the overall trajectory of the contact point track and (ii) friction induced microscopic vibration of the femoral head within the cup (see Fig. 1). Consequently, the sliding distance increased significantly, which increased linear and volumetric wear.

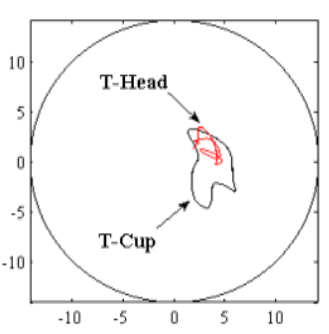

(a)

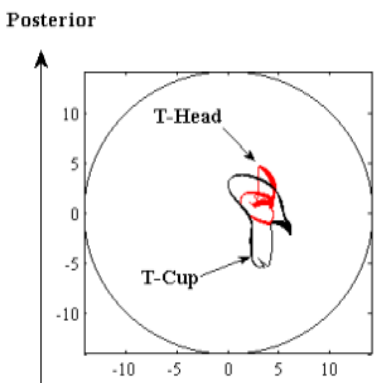

(d)

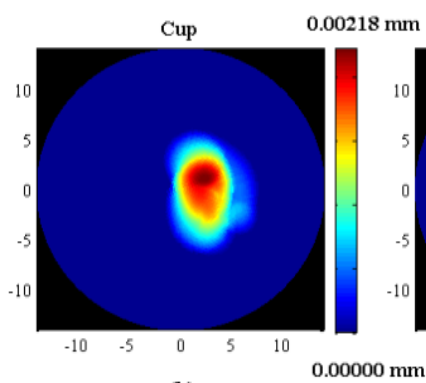

(b)

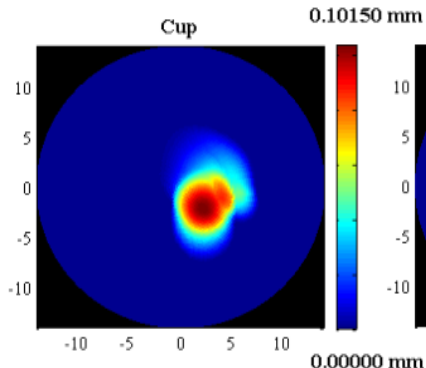

(e)

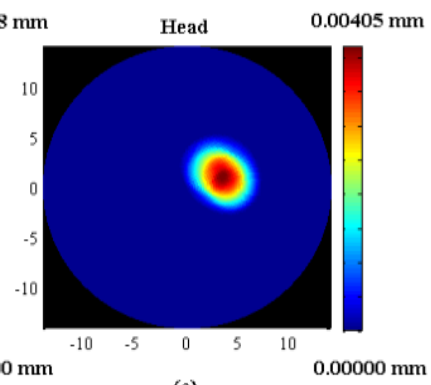

(c)

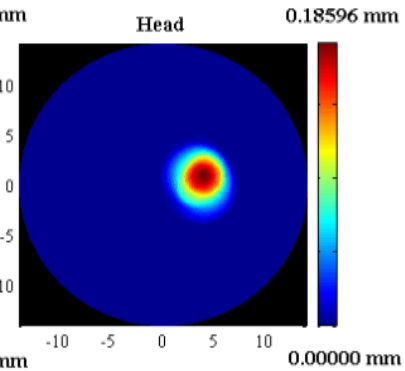

(f)

Medial

Fig. 1. Ceramic-on-ceramic (CoC) hip implant with three-dimensional physiological loading and motion of the human body assuming frictionless where volumetric wear $=0.163 \mathrm{~mm}^{3}$ (top row) and frictional contact where volumetric wear $=6.975 \mathrm{~mm}^{3}$ and $c_{f} / c_{d}=0.1 / 0.065$ (bottom row): (a) and (d) Contact point trajectory on the head and cup, illustrated as T-Head and T-Cup, respectively; (b) and (e) Linear wear depth on the cup; (c) and (f) Linear wear depth on the head.

\section{Conclusions}

A multibody dynamic approach was proposed to integrate the Archard's wear model into our spatial multibody dynamic formulation in order to predict linear and volumetric wear of $\mathrm{CoC}$ artificial hip joints. The gross movement and vibration of the femoral head inside the cup for both frictionless and frictional contact was modelled and wear determined. The alteration of hip implant couple geometries was also taken into account by updating the corresponding surface geome- 
tries as the simulation progressed. The present procedure for very low friction showed very promising results in comparison with the available literature. While previous hip simulators and computational studies have generally underestimated wear rates of artificial hip joints when compared to clinical results, the present article illustrated that friction-induced vibration increased the wear rate significantly. The increase in wear prediction of our model was a result of friction increasing the sliding distance of the femoral head across the cup in both the micro and macro sense, with a wide vibration band and greater overall range of movement of the contact point. The trajectory of the contact point conformed to the wear map on the cup and head surfaces.

Additionally, this study confirmed our hypothesis that friction-induced vibration is the cause of high wear rates observed clinically.

Acknowledgments The first author gratefully acknowledges Macquarie University for International Macquarie University Research Excellence Scholarship (iMQRES) - No. 2010017. The second author expresses his gratitude to the Portuguese Foundation for the Science and Technology under the research project BIOJOINTS (PTDC/EME-PME/099764/2008).

\section{References}

1 Ibrahim, R.A.: Friction-induced vibration, chatter, squeal, and chaos. Part I: Mechanics of contact and friction. Applied Mechanics Review 47(7), 209-226 (1994).

2. Teoh, S.H. Chan, W.H. Thampuran, R.: An elasto-plastic finite element model for polyethylene wear in total hip arthroplasty. Journal of Biomechanics 35(3), 323-330 (2002).

3 Jourdan, F. Samida, A.: An implicit numerical method for wear modelling applied to a hip joint prosthesis problem. Computer Methods in Applied Mechanics and Engineering 198, 2209-2217 (2009).

4 Liu, F. Leslie, I. Williams, S. Fisher, J. Jin, Z.: Development of computational wear simulation of metal-on-metal hip resurfacing replacements. Journal of Biomechanics 41, 686-694 (2008)

5 Boutin, P.: Arthroplastie totale de la hanche par prothése en alumine frittée. Etude expérimentale et premières applications cliniques. Revue Chirurgie Orthopedique 58, 229-46 (1972).

6 Lancaster, J.G. Dowson, D. Isaac, G.H. Fisher, J.: The wear of ultra-high molecular weight polyethylene sliding on metallic and ceramic counterfaces representative of current femoral surfaces in joint replacement. Proceedings of the Institution of Mechanical Engineers, Part H 211, 17-24 (1997).

7 Hegadekatte, V. Huber, N. Kraft, O.: Finite Element Based Simulation of Dry Sliding Wear, Modelling and Simulation in Material Science and Engineering 13, 57-75 (2005).

8 Flodin, A. Andersson, S.: A simplified model for wear prediction in helical gears. Wear 249, 285-292 (2001).

9 Archard, J.F.: Contact and rubbing of flat surfaces. Journal of Applied Physics 24, 981-988 (1953).

10 Mattei, L. Di Puccio, F. Ciulli, E.: A comparative study of wear laws for soft-on-hard hip implants using a mathematical wear model. Tribology International 63, 66-77 (2013). 
11 Saikko, V. Calonius, O.: Slide track analysis of the relative motion between femoral head and acetabular cup in walking and hip simulator. Journal of Biomechanics 35(4), 455-464 (2002).

12 Sariali, E. Stewart, T. Jin, Z. Fisher, J.: Three-dimensional modelling of in vitro hip kinematics under micro-separation regime for ceramic on ceramic total hip prosthesis: An analysis of vibration and noise. Journal of Biomechanics 43, 326-333 (2010).

13 Askari, E. Flores, P. Dabirrahmani, D. Appleyard, R.: Study of the friction-induced vibration and contact mechanics of artificial hip joints. Tribology International 70, 1-10 (2014).

14 Askari, E. Flores, P. Dabirrahmani, D. Appleyard, R.: Nonlinear vibration and dynamics of ceramic on ceramic artificial hip joints: a spatial multibody modelling. Nonlinear Dynamics, DOI 10.1007/s11071-013-1215-y.

15 Mattei, L. Di Puccio, F.: Wear simulation of metal-on-metal hip replacements with frictional contact. Journal of Tribology 135(2), (2013).

16 Nikravesh, P.E.: Computer-Aided Analysis of Mechanical Systems, Prentice-Hall, Englewood Cliffs, NJ (1988).

17 Uddin, M.S. Zhang, L.C.: Predicting the wear of hard-on-hard hip joint prostheses. Wear 301, 192-200 (2013).

18 Dorlot, J.M.: Long-term effects of alumina components in total hip prostheses, Clinical Orthopaedics and Related Research 282, 47-52 (1992).

19 Mittelmeier, H. Heisel, J.: Sixteen years' experience with ceramic hip pros- theses. Clinical Orthopaedics and Related Research 282, 64-72 (1992).

20 Stewart, T. Nevelos, J. Tipper, J. Insley, G. Streicher, R. Ingham, E. Fisher, J.: Long term simulator studies of alumina ceramic/ceramic hip joints with swing phase micro-separation; analysis of wear and wear debris generation. In: Combined Orthopaedic Research Societies Meeting, Rhodes, Greece (2001).

21 Affatato, S. Bersaglia, G. Foltran, I. Emiliani, D. Traina, F. Toni, A.: The influence of implant position on the wear of alumina-on-alumina studied in a hip simulator. Wear 400-405 (2004).

22 Affatato, S. Traina, F. De Fine, M. Carmignato, S. Toni, A.: Alumina-on-alumina hip implants: A Wear study of retrieved components. Journal of Bone and Joint Surgery 94-B, 3742 (2012). 